\title{
Clinical Stage II Retinoblastoma AJCC v8
}

National Cancer Institute

\section{Source}

National Cancer Institute. Clinical Stage I/ Retinoblastoma A/CC v8. NCI Thesaurus. Code C140753.

Stage II includes: cT4a, cNO, cMO, Any H. cT4a: Radiologic evidence of retrobulbar optic nerve involvement or thickening of optic nerve or involvement of orbital tissues. cNO: No regional lymph node involvement. cM0: No signs or symptoms of intracranial or distant metastasis. HX: Unknown or insufficient evidence of a constitutional RB1 gene mutation. H0: Normal RB1 alleles in blood tested with demonstrated high-sensitivity assays. H1: Bilateral retinoblastoma, retinoblastoma with an intracranial primitive neuroectodermal tumor (i.e., trilateral retinoblastoma), patient with family history of retinoblastoma, or molecular definition of a constitutional RB1 gene mutation. (AJCC 8th ed.) 